\title{
$\mathrm{X}$.
}

Aus der chirurgischen Abteilung des Auguste Viktoria-Krankenhauses vom Roten Kreuz in Neu-W eißensee bei Berlin. Dirigierender Arzt: Dr. O. Bod e.

\section{Zur Diagnose und Prognose der Rückenmarksverletzungen.}

Ein Fall von Luxation mit Fraktur des Epistropheus.

$$
\text { Von }
$$

Dr. Richard Bernstein,

Oberarzt beim Garde-Fussartillerie-Regiment, vordem 1. Assistenzarzt des Krankenhauses.

(Mit 3 Abbildungen.)

Die nachstehend veröffentlichten Krankheitsfälle haben das miteinander gemeinsam, daß sie weniger diagnostische als prognostische Schwierigkeiten darboten. Daß sie verhältnismäßig seltene Verletzungen darstellen, dürfte ebenfalls die Veröffentlichung rechtfertigen, für deren Erlaubnis ich meinem hochverehrten früheren Chef, Herrn Dr. Bode, meinen ganz ergebensten Dank ausspreche.

In dem zuerst zu erörternden Falle handelte es sich um einen 21 Jahre alten Tischler. Derselbe war bei Ringübungen in einem Athletenklub dadurch verunglückt, daß er der Länge nach auf eine Matratze geworfen wurde. Er wurde sofort in das Krankenhaus gebracht, wo folgender Befund erhoben wurde:

Kräftiger Mann von gutem Ernährungszustand. Klagt uber Schmerzen im unteren Teile der Halswirbelsäule und zu beiden Seiten derselben.

Mäßige Schwellung der Weichteile in Höhe des siebenten Halswirbels, der auf Beklopfen etwas schmerzhaft ist. Kopf und Nacken vollkommen frei aktiv und passiv beweglich.

Der linke Arm wird in Beugestellung gehalten. Es besteht eine Lälımung sämtlicher vom Nervus radialis versorgten Muskeln (einschließlich des M. triceps). An der Streckseite des Vorderarms ist das Empfindungsvermögen herabgesetzt, es besteht hier Gefühl von Taubheit.

Am rechten Arm besteht eine Lähmung der Fingerstrecker, sowie. dieselbe Empfindungsstörung wie links. 
Am Kopf, am Rumpf und an den unteren Gliedmaßen bestehen weder Lähmungen noch Empfindungsstörungen; die Reflexe zeigen bis auf eine Steigerung der Patellarreflexe nichts Besonderes.

Es handelte sich nun fürs erste darum, Ort und Art der Verletzung festzustellen. Da durch ein Trauma beide Radialnerven, wenn auch nicht in ganz gleicher Weise, betroffen waren, so mußte es mit größter Wahrscheinlichkeit da geschehen sein, wo beide Nerven, bezw. die zu ihnen gehörigen zentralen Bahnen, einander am nächsten liegen; durch diese Annahme, sowie durch die in Höhe des 7. Halswirbels bestehende Schwellung, kam man dazu, die Verletzung auf die drei letzten Zervikalsegmente zu verlegen. Daß es sich um eine Verletzung dieser Segmente, d. h. des Rückenmarks selbst und nicht um eine solche der bereits aus dem Rückenmark ausgetretenen Wurzeln handelte, ließ sich aus dem Fehlen jeglicher Reizerscheinungen auf motorischem wie auf sensibelem Gebiete schließen, da alle Autoren wohl darin übereinstimmen, letztere als charakteristisch für Meningealverletzungen, ihr Fehlen als ebenso charakteristisch für Markverletzungen anzusehen. Ebenso sprach die Steigerung der Patellarreflexe für eine Markverletzung.

War somit die Verletzung auf die graue Substanz der drei letzten Zervikalsegmente lokalisiert, so war es wesentlich schwieriger, die Art derselben festzustellen. Die beiden in Erwägung zu ziehenden Möglichkeiten waren die einer Blutung in die Substanz des Rückenmarks und die einer Erschütterung des Rückenmarks. Bekanntermaßen stehen die meisten Autoren gegenwärtig auf dem Standpunkt, die reine Commotio spinalis, ohne nachweisbare organische Veränderungen des Rückenmarks entweder als überaus selten zu bezeichnen (Oppenheim, Sachs und Freund, von Leyden) oder ihr Vorkommen überhaupt zu bestreiten (Kocher, Stolper). Ohne auf die prinzipielle Frage einzugehen, möchte ich für den in Rede stehenden Fall annehmen, daß es sich mit großer Wahrscheinlichkeit um eine Blutung in die graue Substanz, nicht um eine Rückenmarkserschütterung gehandelt hat. Wenn etwas für letztere sprechen könnte, so war es nur der überaus rasche Verlauf, den die weitere Entwicklung der Krankheitserscheinungen nahm.

Es bestand nämlich bereits am Morgen nach dem Unfall, d. b. 9 Stunden nach demselben, keine motorische Lähmung mehr, die Sensibilitätsstörungen waren ebenfalls im raschen Rückgange begriffen, und die anfangs noch bestehende Schwäche der gelähmt gewesenen Muskeln verschwand so schnell, dab der Verletzte drei Wochen nach dem Unfall als geheilt und arbeitsfähig das Krankenhaus verlassen konnte. 
Aber trotz dieses raschen Rückganges aller Krankheitszeichen möchte ich dieselben nicht auf eine bloße Erschütterung des Rückenmarks zurückführen. Es erscheint mir zu wenig wahrscheinlich, daß die Erschütterung die eine Stelle so stark getroffen haben sollte, daß die Funktion ganzer Nervenzellengruppen vollständig aufgehoben wurde, während die übrigen Gebiete des Rückenmarks keinerlei Funktionsstörungen zeigten. Viel leichter kann ich mir vorstellen, daß ein durch die Verletzung entstandener Bluterguß, vermöge der ausgiebigen Versorgung der grauen Substanz mit Blut- und Lymphgefäßkapillaren, rascher aufgesaugt worden sei, als wir es von anderen Blutergüssen, z. B. den unter der Haut gelegenen, gewohnt sind.

Wenn wir nun noch nach einer Erklärung für das annähernd symmetrische Auftreten der Lähmungserscheinungen suchen, so würden wir dieselbe darin zu finden haben, dal das extravasierte Blut sich von der Gegend der grauen Kommissur aus nach beiden Seiten hin verbreitet hat. Diese Gegend, das Querstück des von der grauen Substanz im Querschnitt gebildeten $H$, ist diejenige, in die Goldscheider und Flata u (Zeitschr. f. klin. Medizin 1897, Bd. 31) die Einspritzungen machten, bei denen sie die größte Querausbreitung in der grauen Substanz erzielten, indem die Flüssigkeit auch auf die andere Seite sich den Weg bahnte. Gerade in dieser Gegend aber tritt, wie z. B. aus der Zieh en schen Abbildung dentlich hervorgeht, die Arteria fissurae anterioris in die graue Substanz des Rückenmarks hinein; erfolgte ihre Verletzung an dieser Stelle, so konnte sich das Blut aus einer Rißstelle in beide Vorderhörner, teilweise auch noch in beide Hinterhörner hinein ergießen und dort so lange die Funktion der Zellen der Radialiswurzeln durch Druck aufheben, bis es zur Resorption gelangt war. Ich möchte in dieser Auffassung des Krankheitsbildes eine klinische Bestätigung der anatomischen Untersuchungen und der erwähnten Versuche erblicken.

Der folgende Fall gibt Anlaß, auf die Frage der Differentialdiagnose zwischen Verletzung des unteren Endes des Rückenmarks und einer solchen der Cauda equina, sowie auf die prognostische Bedeutung derselben einzugehen.

Ein mäßig kräftiger Brauereiarbeiter, 30 Jahre alt, war seinen Angaben zufolge in einem Keller bei der Arbeit hingestürzt und mit dem Rücken gegen eine Faßkante anfgeschlagen. Er will sofort heftige Schmerzen im Rücken verspürt haben und konnte angeblich weder gehen noch stehen. Er kam leider erst nach zwei Tagen in das Krankenhaus, so daß über den Zustand unmittelbar nach der Verletzung keine genauen Angaben vorliegen. Bei der Aufnahme bestand uber dem 2. und 3. Lenden- 
wirbel leichte Schwellung der Weichteile und Verfärbung der Hant, sowie ziemlich heftiger Schmerz beim Betasten dieser beiden Dornfortsätze. Das rechte Bein konnte im Huftgelenk ab- und adduziert, nur in geringem Grade gebeugt werden. Im Knie und in den Fußgelenken war aktive Beugung nicht möglich, bei passiver Beugung erfolgte sofort wieder Streckung. Das Empfindungsvermögen war am Untersehenkel und am Fuß vollkommen aufgehoben, an der Beugeseite des Oberschenkels von der Mitte ab nach abwärts, an der Streckseite im unteren Drittel. In den oberen Teilen des Oberschenkels, sowie am Damm, Penis und Skrotum war dasselbe sehr beträchtlich herabgesetzt. Der Temperatursinn war in gleicher Weise verändert. Die Reflexe konnten nicht ausgelöst werden. - Am linken Bein, sowie am übrigen Körper waren keinerlei Störungen nachweisbar.

Zeitweise traten schießende Schmerzen längs der Beugeseite des rechten Beins auf.

Im Befund trat acht Tage hindurch keine Änderung auf; am zwölften Tage nach dem Unfall war bereits aktive Beugung und Streckung im Fuß- und in den Zehengelenken möglich, am sechzehnten Tage auch im Kniegelenk. Die zu dieser Zeit bestehenden Sensibilitätsstörungen entsprachen fast genau dem Gebiete der ersten, zweiten und dritten Sakralwurzel des Seifferschen Schemas; sie gingen in den nächsten Tagen in der Weise zurück, daß das Gebiet der Sakralwurzel 3 zuerst, das der Sakralwarzel 1 zuletzt sich besserte. In gleicher Weise waren die den beiden ersten Sakralsegmenten entsprechenden Motilitätsstörungen fruher zurüekgegangen als die auf das fünfte Lumbalsegment zurückzuführenden. Die elektrische Erregbarkeit der gelähmt gewesenen Muskeln war während des ganzen Verlanfes normal, Entartungsreaktion nie festzustellen.

Als der Verletzte aus anderen Gründen sieben Wochen nach dem Unfall das Krankenhaus verlassen mußte, bestanden keine anderen Störungen als eine vollständige Anästhesie in einem etwa handbreiten Streifen längs der Beugeseite des Oberschenkels; weitere fünf Wochen später hatte auch diese sich soweit gebessert, daß Nadelstiche, wenn auch schwächer als links, empfunden wurden.

An dem hier mitgeteilten Falle ist zunächst der Umstand, daß nur eine Seite befallen ist, bemerkenswert, da nach von Leyden und Goldscheider Läsionen der Cauda equina ebenso wie diejenigen des untersten Markendes meist symmetrisch sind.

Für die Differentialdiagnose dieser beiden Läsionen geht die Meinung der meisten Autoren dahin, daß sie sehr schwierig, vielfach unmöglich ist. Wollten wir in unserem Falle mit Sachs und Freund deshalb eine Markverletzung ausschließen, weil „unterhalb der direkt getroffenen Stelle gelegene Centra nicht affiziert sind ${ }^{\mu}$, d. h. weil zwar die von den oberen Sakralsegmenten versorgten Unterschenkelmuskeln, nicht aber die von den unteren Sakralsegmenten versorgten Blasen- und Mastdarmmuskeln gelähmt sind, so widerspricht dem nicht nur unser erster Fall, sondern auch zahlreiche andere in der Literatur mit- 
geteilte Beobachtungen. Auch darf das Fehlen des Brown-Sequardschen Typus, d. h. die Gleichseitigkeit der motorischen und sensibelen Lähmung, nicht als Grund gegen die Annahme einer Markläsion herangezogen werden, indem oppenheim darauf hinweist, daß, „wenn die halbseitige Affektion tief im Lendenmark resp. Sakralmark sitzt, die Lähmung und im wesentlichen auch die Gefühlsstörung das Bein der entsprechenden Seite betrifft, da in dieser Höhe naturgemäß nur wenige sensibele Wurzeln auf die andere Seite gelangt sind".

Andererseits dürfen wir nicht durch das in unserem Falle festgestellte Fehlen der Entartungsreaktion „die Wagschale für den Sitz im Mark sinken lassen" (von Leyden und Goldscheider).

Vergleichen wir die von Wag $g^{\circ}$ er und Stolper für die partielle Markläsion auf der einen und für die extramedulläre Blutung im Wirbelkanal auf der anderen Seite gegebenen Symptome, so entspricht unser Fall weder ganz der einen, noch ganz der anderen Symptomengruppe; immerhin aber scheint das Auftreten der schielenden Schmerzen, sowie die spastische Form der Lähmung eine zum mindesten erhebliche Beteiligung der Cauda equina zu erfordern, wiewohl zuzugeben ist, dali bei dieser noch schwerer als beim Rückenmark die Einseitigkeit der Affektion sich erklären läßt. $\Lambda \mathrm{m}$ sichersten gehen wir wohl, wenn wir gleichzeitige Verletzung von Mark und Cauda annehmen, und als Ort der Verletzung der letzteren ihre Austrittsstelle aus dem Mark.

Daf wir im vorliegenden Falle eine Blutung als Ursache der nervösen Störungen annahmen, ergab sich naturgemäl erst aus dem Verlauf, aus dem verhältnismäßig schnellen Rückgang der Lähmungsund Reizungserscheinungen. Im Anfang lag zu dieser Annahme keine Veranlassung vor; es gab keine Möglichkeit, eine Zerquetschung der Rückenmarkshälfte oder der Caudastränge etwa durch einen Wirbelsplitter auszuschließen, und dementsprechend eine so günstige Prognose zu stellen, wie sie schließlich ex eventu zu stellen war.

Während in den beiden bisher mitgeteilten Fällen der Verlauf sich günstiger gestaltete, als sich anfangs erwarten lieb, bietet der folgende Fall die Eigentümlichkeit, dal die gemäl der Art der Versetzung zu erwartende Beteiligung des Rückenmarks zunächst vollkommen fehlte und erst zu einer Zeit auftrat, wo sie kaum noch zu befürchten war.

Die Yerletzung, um die es sich handelt, war eine Luxation des Epistropheus, die bei einem 18jährigen Kutseher dadurch zustande gekommen war, daß ihm das Schwungrad einer Sägemaschine gegen die linke Hals- 
seite gefallen war. Bei der Aufnahme war der Kopf etwa um $40^{\circ}$ nach links gedreht und etwas vorwärts gebeugt, wie aus den Abbildungen 1 und 2 hervorgeht. Bewegungen waren nur im Sinne der Drehung in ganz geringer Exkursion ausfuhrbar. Dicht über der Stelle, wo der dritte Halswirbeldorn zu erwarten war, fuhlte man rechts neben der Mittellinie einen abnormen Knochenvorsprung, links eine flache Einsenkung. Das von der Seite her aufgenommene Röntgenbild, Abbildung 3 1), dessen Deutung durch die mit der Aufnahme verbundenen Schwierigkeiten nicht eben leicht war, zeigte folgende auffallende Punkte:

1. Der Dornfortsatz des Epistropheus liegt im Vergleich mit den folgenden Dornfortsätzen auffallend dicht unter der Haut, außerdem nicht in derselben Sagittalebene wie sie, da sein Schatten intensiver und breiter erscheint.

2. Die sagittale Achse des Atlas scheint mit der des Epistropheus-

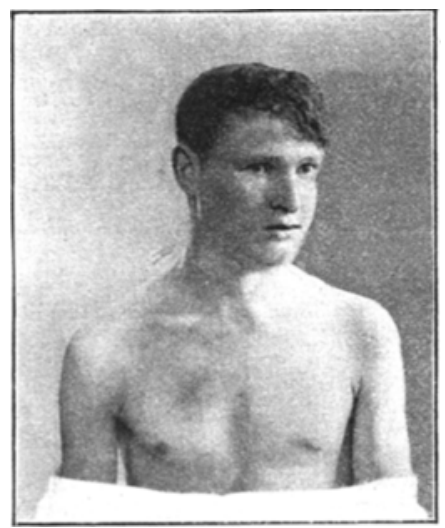

Fig. 1.

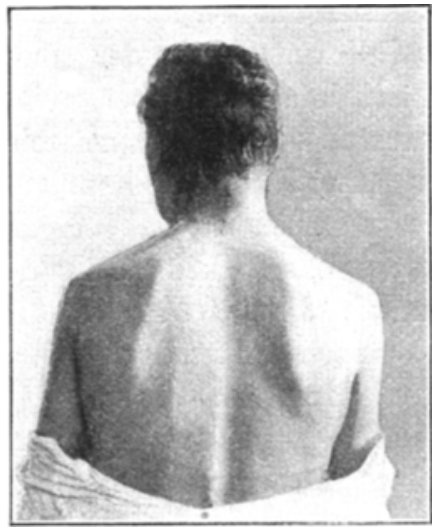

Fig. 2.

körpers einen abnorm spitzen Winkel zu bilden; man gewinnt den Eindruck, als ob die rechte Seitenfläche des Epistropheuskörpers von dem rechten Seitenbogen des Atlas thberlagert wird. Leider schiebt sich bier der Schatten des rechten Warzenfortsatzes vor und überdeckt gerade die Gegend der Gelenke zwischen Atlas und Epistrophens.

Die genannten Symptome ließen die Diagnose auf eine Luxation des Epistropheus gegen den Atlas als sehr wahrscheinlich erschein en. nicht.

Lähmungserscheinungen irgendwelcher Art bestanden

Da bereits vier Wochen seit dem Unfall vergangen waren, während deren der Verunglttekte erst in einem anderen Krankenhause, dann zu Hause behandelt worden war, wurde von Repositionsversuchen abgesehen.

1) Die Lichtbilder sind im Berliner medico-mechanischen Institut (San.-Rat Dr. Schütz) angefertigt worden. 
Wegen einer vor dem Kieferwinkel bestehenden Eiterung konnte auch eine Feststellung des Kopfes und des Halses nicht vorgenommen werden. Bis zum 71. Tage nach dem Unfall blieb der Verletzte völlig beschwerdefrei; daß keinerlei Störung seitens des Rückenmarks vorlag, geht daraus hervor, daß er sich eines Tages - natillich ohne Erlaubnis - durch Tragen schwerer Balken am Bau einer Döckerschen Baracke beteiligte. Erst am 72. Tage zeigten sich nervöse Erscheinungen, die mit dem rechten Arm anfangend innerhalb von vierzehn Tagen auf das rechte Bein, den linken Arm, das linke Bein, Blase und Mastdarm in der angefuhrten

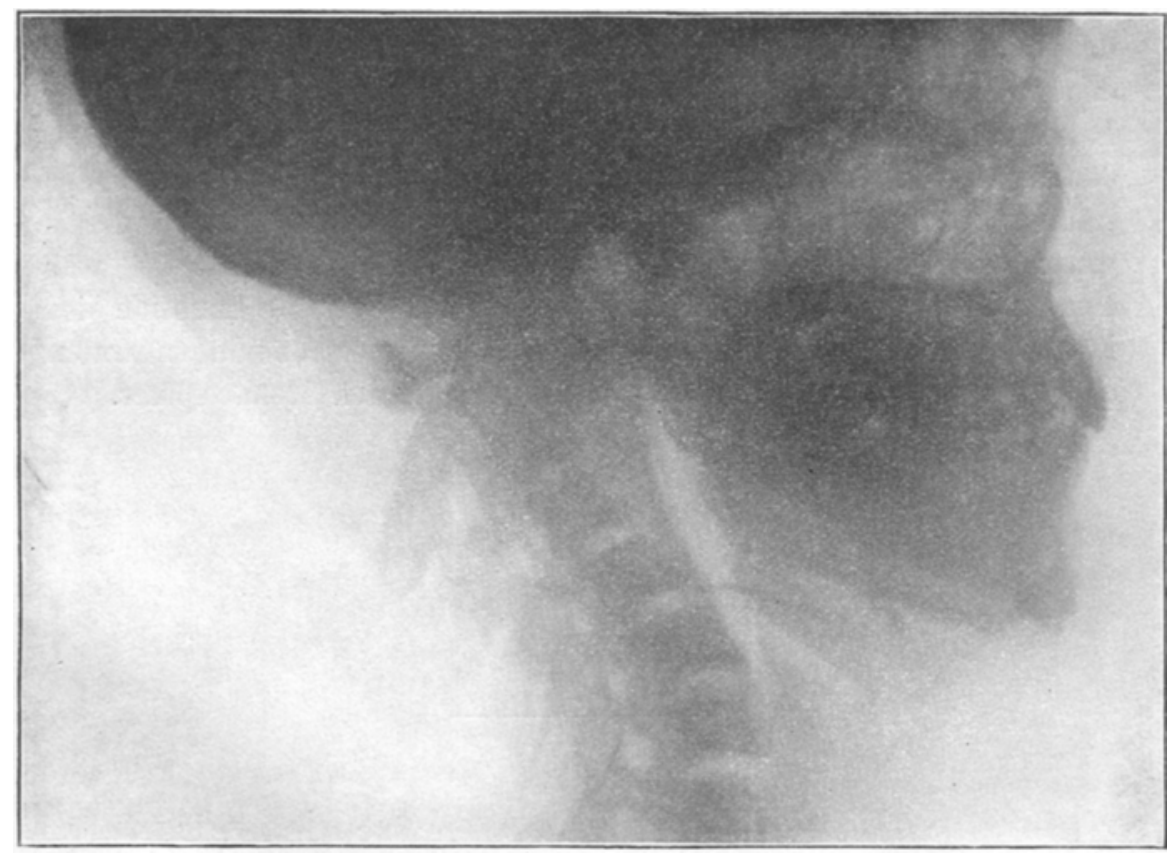

Fig. 3.

Reihenfolge übergingen und vorzugsweise das motorische Gebiet in Form von spastischer Lähmung betrafen; Patellarreflexe, Fußklonus waren gesteigert, Pupillen reagierten gut. Gegen Ende traten heftige Schmerzen in den Gliedern, Phrenicuslähmung und Decubitus auf. Der Tod erfolgte am 101. Tage nach dem Unfall. Die Sektion ergab eine Drehungsluxation im Atlas-Epistropheusgelenk in der Art, daß die rechte Gelenkfläche des Epistropheus sich nach vorn verschoben hatte; außerdem fand sich an der Innenfläche des Epistropheus-Dornfortsatzes eine Knochenwucherung (Kallus), die offenbar durch ihr Wachstum allmählich stärker auf das Rilckenmark gedrückt hatte. Die genauere Untersuchung des Rückenmarks steht noch aus; jedoch ist von ihr wenig zu erwarten, da die 
182 X. Bernstein, Zur Diagnose und Prognose der Rückenmarksverletzungen.

Sektion, die im Hochsommer stattfand, durch die unvermeidlichen gerichtlichen Schwierigkeiten erst mehrere Tage nach dem Tode vorgenommen werden konnte.

Zweierlei erscheint an diesem Falle, klinisch sowohl wie vom Standpunkt des Gutachters, bemerkenswert: Das völlige Fehlen medullarer Erscheinungen während eines so langen nach der Verletzung verstreichenden Zeitraumes und das allmählich erfolgende Einsetzen derselben. Soweit ich die Literatur durchsehen konnte, sind nur zwei ähnliche Beobachtungen veröffentlicht worden, die Wagner und Stolper mitteilen. Der eine Fall, von Preiß aus dem Jahre 1792, betrifft allerdings eine Verrenkung des dritten gegen den zweiten Halswirbel, stimmt aber insofern sehr gut zu unserem Falle, als auch hier die ersten Lähmungserscheinungen erst nach 60 Tagen, der Tod am 101. Tage erfolgte; auch das Auftreten der Lähmungen geschah in gleicher Reihenfolge, nur bestand merkwürdigerweise keine Blasenlähmung. Der andere Fall (Costes 1852) war eine mit Bruch des Zahnfortsatzes verbundene partielle Luxation des Epistropheus gegen den Atlas; die Lähmungserscheinungen traten erst nach vier Monaten auf, der Tod einen halben Monat später. 